\title{
Einige Bemerkungen zu der Arbeit von Näcke, Zwei sexologisehe Themen: 1. Die Zeugung im Rausche und ihre schädlichen Folgen
}

in Bd. XI, S. 218 dieser Zeitschrift.

Von

Dr. R. Fels (Lennep).

(Eingegangen am 18. Januar 1913.)

Durch einen Zufall habe ich die betreffende Arbeit bis heute übersehen, ich möchte jedoch im Interesse der Bearbeitung des Themas nachstehende Dinge nicht ungesagt sein lassen.

Näcke bezeichnet seine Ausführungen als kritische und eben vom Standpunkte der Kritik aus habe ich Bedenken gegen seine Darstellung.

Zugegeben, daß der Begriff $\mathrm{R}$ a u sc $\mathrm{h}$ sich lediglich auf die psychischen Erscheinungen der Alkoholintoxikation bezieht, so müßte Näcke bekannt sein, daß es eine alte Forderung der Abstinenten (so richtig statt „Abstinenzler", ebenso wie Sexologe und nicht Sexologler) ist, in foro und anderweit statt des Ausdruckes Rausch die Bezeichnung unter Alkoholwirkung stehend zu wählen. Auch Näcke gibt ja schließlich den Ausdruck akute Alkoholintoxikation, verlangt jedoch im nächsten Absatze, der Rausch müsse beschrieben werden.

Schreck und Sorgen und geringe Unpäßlichkeiten der Eltern werden von Näcke ohne weiteres als Minderwertigkeiten angenommen, die das Produkt des Beischlafes im ungünstigen Sinne beeinflussen. Wo aber sind dafür Beweise, die der Kritik Stich halten? Nach Näckes Meinung müßten die „Hochzeitkinder" besonders gefährdet sein, daß aber der unter der Alkoholwirkung des Hochzeitsfestes stattfindende erste Beischlaf in den allerseltensten Fällen zur Befruchtung führt, müßte Näcke doch bekannt sein. Würde behauptet, die Minderwertigkeit der Erstgeborenen sei alkoholisch bedingt, so wäre Näcke der erste, der darauf hinwiese, daß die Erstgeborenen in einer the in kaum einem Falle auch nur mit Wahrscheinlichkeit einem einmaligen Beischlafe entstammten.

Die darauf folgende Kritik der Fälle Holitschers erscheint vom kritischen Standpunkte aus in besonderem Lichte; wenn man folgendes 
beachtet. Holitscher schreibt in seiner Veröffentlichung von 1909: „Nicht nur die Eltern, auch die Großeltern und ein Teil der Aszendenz sind mir sehr gut bekannt, irgendwelche Entartungserscheinungen kommen in der Familie nicht vor." Näcke bemerkt dazu: „Auch hat Holitscher wohl kaum die Eltern, Großeltern usw. genau psychiatrischpsychologisch untersucht.“ Näcke sagt ferner: „Endlich hat er nicht bewiesen, daß es sich nur um einen einzigen Coitus handelte." Holitscher schreibt wörtlich: „Da natürlich die Hoffnung bestand, das eine Mal werde vielleicht ungestraft bleiben, so wurden die gewohnten Vorsichtsmaßregeln auch danach wieder angewendet, bis sich die untrüglichen Symptome der Gravidität einstellten, die Tatsache der Zeugung während der Alkoholvergiftung ist darum durchaus feststehend."

$\mathrm{Da} B$ die statistischen Massenuntersuchungen für das Problem nur bedingten Wert haben, ist den Forschern, die zufällig auch Alkoholgegner sind, auch bekannt, sie weisen auch auf die Tatsache hin, daß Bezzolas Zahlen schon aus rein rechnerischen Gründen nicht eindeutig beweisend sind. Aber Bezzola bringt doch auch die Konzeptionszahlen der Normalgeburten, mithin das von $\mathrm{Näc}$ ke gewünschte Material. Den Einwand Holitschers a. a. O., daß es gerade die sonst solide Lebenden sind, die in Festzeiten exzedieren, während die Psychopathen und Trinker dieser nicht erst bedürfen, läßt Näcke ebenso unerwähnt wie unwiderlegt.

Nicloux und Renaut werden zitiert, aber die Forscher, die zu entgegengesetzten Ergebnissen kamen, werden leider nicht namentlich angeführt. Die Zahlenwerte Nicloux' hätten aber Näcke darauf hinweisen können, daß sein Ausdruck von der ,,doch immerhin geringen Alkoholmenge" in Holitschers erstem Falle nicht berechtigt war. Denn 7,5 l Bier zu 4\% ergibt doch bei einem Manne von angenommen $75 \mathrm{~kg}$ eine Alkoholmenge von $4 \mathrm{ccm}$ pro kg. Das ist aber eine beachtenswerte toxische Dosis. Näc ke fragt weiter: ,Wie lange blieb der Alkohol (im Hoden) unzersetzt?" Auch hierüber finden sich experimentelle Ergebnisse bei Nicloux.

So exakt ferner die Forderung Näckes erscheint, der Alkohol müsse mikroskopisch nachgewiesen sein, um die Blastophtorie zu beweisen, so übersieht er dabei die Möglichkeit von Strukturänderungen, die durch veränderten Chemismus der Samenflüssigkeit zum Beispiel gesetzt werden könnten, und die, alkoholiseh bedingt, zur Blastophtorie führen könnten, ohne daß der Alkohol in den Spermatozoen chemisch nachznweisen ist.

Aus seinen Darlegungen hat $\mathrm{Näc}$ ke ,ersehen, daß nur in sehr großen Ausnahmefällen die Rauschzeugung schädlichen Einfluß hat". Diesen Beweis hat Näcke aber nicht erbracht, weil er zur Zeit nicht zu erbringen ist, wenn man sämtliche methodischen Forderungen Näckes 
erfüllen will. Näcke hat vielmehr lediglich festgestellt, daß die Zahl der bisher veröffentlichten Individualfälle eine sehr geringe ist. Leider wird dies bei der mangelhaften Kenntnis des Alkoholproblems unter den Ärzten wohl noch für längere Zeit so bleiben.

Verfehlen möchte ich nicht, diejenigen, die das Problem der wissenschaftlichen Kritik interessiert, auf die meines Wissens erstmalige Bereicherung einer wissenschaftlichen Kritik durch Stellen aus geistreichen Privatbriefen hinzuweisen, wie sie Näcke in einer Anmerkung bringt. Die Formen der wissenschaftlichen Kritik haben dadurch eine nennenswerte Bereicherung erfahren und bei reicher Anwendung dieser Methode wird zweifellos der kritische Geist in der Medizin immer reifer werden und das verdanken wir dann Näcke. 